\title{
Carriage and serotype distribution of Streptococcus agalactiae in third trimester pregnancy in southern Ghana
}

\author{
Hans-Christian Slotved ${ }^{1 *}$ D, Nicholas T. K. D. Dayie ${ }^{2}$, Josephine A. N. Banini ${ }^{2}$ and Niels Frimodt-Møller ${ }^{3}$
}

\begin{abstract}
Background: The aim of this study was to determine the prevalence of Streptococcus agalactiae (group B streptococci, GBS) among healthy, pregnant women attending antenatal care at different study sites in the Greater Accra Region, Ghana.

Methods: Between 2010 and June 2013, recto-vaginal swab samples were collected from pregnant women attending antenatal care from two study sites in southern Ghana. The samples were collected within 35 and 37 weeks of the gestation period. These were inoculated into Todd-Hewitt broth followed by sub-culturing onto a sheep-blood agar plate. Identification was performed on a single subcultured colony. Gram staining was performed, and isolates were evaluated for beta-haemolytic reactions. Furthermore, the isolates were serotyped using the GBS latex serotyping kit.

Results: The carriage rates were found to be $25.5 \%$ (95\% Cl: 19.6-32.1) to 28.0\% (95\% Cl: 21.9-34.8) for the two collection sites. The most common serotypes were serotypes VII and IX. The data showed that women below 20 years of age or above 30 years of age have a significantly $(p=0.037)$ higher risk of carrying GBS compared to women from the age group of 20 to 30 years.

Conclusions: The findings of this study revealed that prevalence of GBS colonization in pregnant women in Greater Accra region is high and comparable to rates observed in South Africa and Western countries. The most prevalent serotypes were serotypes VII and IX, which have not been observed before in West Africa.
\end{abstract}

Keywords: Streptococcus agalactiae, Ghana, Carriage, Pregnancy

\section{Background}

Streptococcus agalactiae (group B streptococci, GBS) is a well-known human opportunistic pathogen primarily causing infections in newborns, the elderly and invasive disease in adults with underlying medical conditions [1]. The pathogenesis of the disease in neonates is generally described in two stages: i) Early-onset disease (EOD) which by definition occurs in the neonate during the first six days of life, or ii) late-onset disease (LOD) which is defined as occurring from seven days of life and can develop up to three months of age $[1,2]$. Possible clinical manifestations of GBS infection in neoantes are

\footnotetext{
*Correspondence: hcs@ssi.dk

'Department of Bacteria, Parasites and Fungi, Statens Serum Institut,

Artillerivej 5,-2300 Copenhagen, DK, Denmark

Full list of author information is available at the end of the article
}

sepis, meningitis and pneumonia [3]. Among adults, GBS is also associated with invasive infections, particularly in elderly persons with underlying medical conditions [1]. The human GBS are currently divided into ten serotypes based on serotype specific antigens and are designated as Ia, Ib, II, III, IV, V, VI, VII, VIII, and IX [1].

Despite advances in medical practice in developed countries, studies have shown that GBS is still considered a life-threatening pathogen; however, antenatal screening of pregnant women between weeks 35 and 37 and subsequent administration of antibiotic prophylaxis to carriers has led to a reduction in GBS diseases in some countries among neonates and women in recent years [4]. In a surveillance study from USA describing the EOD and LOD from 1990 to 2010, the impact of implementation of different GBS disease prevention 
strategies resulted in a decline in the EOD, although the LOD remains the same [3]. This is, however, not the case in the developing countries and particularly in Africa [1, 4], where GBS is still one of the the leading cause of neonatal diseases. A particular problem with recognizing GBS as an important cause of disease in infants in Africa is the lack of data on the prevalence of colonization in pregnant women in most countries [1]. In recent years, carriage studies from African countries have emerged, showing GBS serotype distribution data from countries such as Gabon, Ethiopia, the Democratic Republic of the Congo, and Zimbabwe [4-7]. In addition, a recent publication from the Central Region of Ghana showed the first carriage rate of GBS among pregnant women [2].

The aim of this study was to determine the prevalence and serotype epidemiology of GBS isolated from pregnant women in southern Ghana.

\section{Methods}

\section{Study design and study site}

This study was carried out in the Greater Accra Region. This region is situated in the southern part of Ghana and has a high population density of $15.8 \%$.

The study was carried out between May 2012 and June 2013 in the Greater Accra region involving two health facilities. Samples were collected from Mamprobi Polyclinic, Accra, and the Dangme West District Hospital, Dodowa, and the laboratory work was performed at the National Public Reference Laboratory, Korle-Bu Teaching Hospital. The Mamprobi Polyclinic is situated in the Ablekuma Sub-metro, which is the largest of the six sub-metros in Accra with a total population of 691,364. The Dangme West District Hospital serves as a major referral center for the people of the Dangme West District, which has a population of 136,622.

\section{Inclusion criteria}

All pregnant women in the last trimester of their pregnancy (35 to 37 weeks of gestation) who consented to participate in the research were included in the studies.

\section{Collection of samples}

Four hundred third trimester pregnant women between 35 and 37 weeks of gestation attending the routine antenatal clinics at the Mamprobi Polyclinic, Accra, and Dangme West District Hospital, Dodowa, were included in the study. A total of 400 vaginal and rectal swab samples were collected between August 2012 and March 2013. Thus, samples from 200 pregnant women were collected from each study site.

\section{Swab sample collection}

Two sterile cotton swab sticks were used to take two different swabs from each person: 1) A swab from the lower vagina and 2) a swab from the rectum. The swabs were collected either by qualified health workers or by the subject themselves after providing them with appropriate instructions $[8,9]$.

Questionnaires were used to obtain other demographic information. Information retrieved from the women included age, time of pregnancy, parity, previous abortions, level of education, presence of diabetes mellitus, and urinary tract infection (UTI).

\section{Specimen processing and culturing}

An enriched culture medium (ECM) technique was employed. Immediately after the samples were collected under aseptic conditions, the swab sticks were transferred into a selective enrichment broth medium (Todd Hewitt broth) (oxoid LTD) supplemented with gentamicin $(8 \mu \mathrm{g} / \mathrm{ml})$, and nalidixic acid (Sigma Aldrich, $15 \mu \mathrm{g} / \mathrm{ml}$ ) and $5 \%$ sheep blood to increase the recovery rate of GBS $[8,9]$. The collected samples were transported to the laboratory within three hours of sample collection.

In the laboratory, the inoculated broths were incubated overnight $(18-24 \mathrm{~h})$ at $37^{\circ} \mathrm{C}$. The broths were subcultured onto 5\% sheep blood agar plates (Liofilchem S.R.L. Bacteriology products, Italy) and incubated for 18 to $24 \mathrm{~h}$ at $37{ }^{\circ} \mathrm{C}$ in $5 \% \mathrm{CO}_{2}$. Plates with no growth were reincubated for an extra $24 \mathrm{~h}$.

Plates with colonies were examined for their characteristic colonial morphology. Beta-hemolytic and nonhemolytic colonies were also considered. The suspected colonies were Gram stained (Gram positive) and tested for catalase reaction (catalase negative) using 3\% hydrogen peroxide. The suspected colonies were further tested using Group B latex agglutination kit (Latex agglutination test, Statens Serum Institut, Copenhagen, Denmark) to confirm the identity of GBS. The confirmed GBS were serotyped using the Strep-B-Latex kit (Statens Serum Institut, Denmark). Strains were cultured for $24 \mathrm{~h}$ in Todd Hewitt broth. Ten microlitres from this culture was mixed with specific antisera against serotypes Ia, Ib, and II-IX specific to CPS antigens latex agglutination suspension, and agglutination was read after 5 to $10 \mathrm{~s}$ [10].

\section{Storage}

The isolates were stored at $-80{ }^{\circ} \mathrm{C}$ in a broth containing skimmed milk, tryptone, glucose and glycerol (STGG) [11] at the National Public Reference Laboratory, Korle-Bu.

\section{Data analysis}

All statistical calculations of $p$-values (Either Welch Two Sample t-test or the Chi-square test), odds ratios (OR) 
and confidence intervals $(\mathrm{CI})$ were performed using $\mathrm{R}$ version 3.1.3 for Windows (http://www.r-project.org/).

\section{Results}

\section{Characteristics of study population}

A total of 400 women were enrolled. The characteristics from the two tested sites are presented in Table 1. The two groups showed similar characteristics with regard to mean age, number of abortions, education level and prevalence of urinary tract infections. In addition, no significant differences were found between the two groups except with regards to the number of times a woman had given birth. In Dangme West, the parity was significantly higher $(p=0.01)$ than in Mamprobi. Only two women from Mamprobi Polyclinic had diabetes, and risk assessment was therefore not performed on GBS versus diabetes.

\section{The GBS prevalence for the various sites.}

Of the 200 women from Mamprobi (Table 2), 56 of them were carriers of GBS (28.0\%, 95\% CI: 21.9-34.8), as opposed to 51 women at Dangme West (25.5\%, 95\% CI: 19.6-32.1). One woman from Dangme West was found to carry multiple GBS serotypes (serotype IV and serotype IX).

Of the 56 positive samples from Mamprobi, 44 women had positive GBS vaginal samples, four had both positive vaginal and rectal samples and eight had rectal positive samples only. In Dangme West, 40 women had positive vaginal samples only, two had both vaginal and rectal positive samples, and nine had positive rectal samples only.

Table 2 shows the serotype distribution for both sites. Eight of the ten known GBS serotypes were found in the study, although not equally represented at all four sites. Serotype Ib and VI was not found at the sites. The study showed a serotype distribution in which serotypes VII and IX were the dominant serotypes found at both study sites. At Mamprobi, $42.9 \%$ of the isolates were serotype VII, while $32.1 \%$ were serotype IX. In Dangme West, $38.5 \%$ of the isolates were serotype VII, while $26.9 \%$ were serotype IX.

\section{Association between social demographic factors and GBS colonization \\ Risk factors for Mamprobi}

Age was found to be a risk factor (Table 3). Women below 20 years of age or above 30 years of age have a significantly ( $p=0.037)$ higher risk of carrying GBS compared to women from the age group of 20 to 30 years. Individual risk factors such as parity, abortions, the level of education, and urinary tract infections did not show to have any effect on the risk of carrying GBS.
Table 1 Grouping of the participants from each site

\begin{tabular}{|c|c|c|c|}
\hline & $\begin{array}{l}\text { Mamprobi } \\
\text { (Accra) } \\
N=200 \\
\text { (Range) }\end{array}$ & $\begin{array}{l}\text { Dangme West } \\
\text { (Dodowa) } \\
N=200 \\
\text { (Range) }\end{array}$ & $\begin{array}{l}P \text { values or } \\
\text { Odds ratio } \\
(95 \% \text { confidence } \\
\text { interval) })^{\mathrm{a}}\end{array}$ \\
\hline \multicolumn{4}{|c|}{ Maternal age group } \\
\hline$<20$ & $14^{c}$ & 0 & \\
\hline $20-30$ & 118 & 128 & \\
\hline$>30$ & 67 & 72 & \\
\hline Unknown ${ }^{b}$ & 1 & 0 & \\
\hline Mean age & $27.99(15-42)$ & $28.97(21-44)$ & $P=0.06(-1.98--0.04)$ \\
\hline \multicolumn{4}{|l|}{ Parity } \\
\hline 0 & 43 & 24 & \\
\hline 1 & 56 & 68 & \\
\hline$>1$ & 78 & 108 & \\
\hline Unknown ${ }^{\mathrm{b}}$ & 23 & 0 & \\
\hline Mean parity & $1.5(0-7)$ & $1.9(0-5)$ & $P=0.01(-0.62--0.08)^{*}$ \\
\hline \multicolumn{4}{|l|}{ Abortion } \\
\hline 0 & 114 & 132 & \\
\hline 1 & 30 & 48 & \\
\hline$>1$ & 14 & 20 & \\
\hline Unknown ${ }^{b}$ & 42 & 0 & \\
\hline $\begin{array}{l}\text { Mean } \\
\text { abortion }\end{array}$ & $0.4(0-6)$ & $0.5(0-5)$ & $P=0.33(-0.27-0.09)$ \\
\hline \multicolumn{4}{|l|}{ Education } \\
\hline $\begin{array}{l}\text { None or } \\
\text { Primary }\end{array}$ & 54 & 56 & \\
\hline $\begin{array}{l}\text { Secondary } \\
\text { or more }\end{array}$ & 142 & 144 & $\begin{array}{l}\mathrm{OR}=1.02(0.66-1.59) \\
P=0.921\end{array}$ \\
\hline Unknown ${ }^{\mathrm{b}}$ & 4 & 0 & \\
\hline \multicolumn{4}{|c|}{ Urinary tract infection (UTI) } \\
\hline Yes & 11 & 12 & \\
\hline No & 187 & 120 & $\begin{array}{l}\mathrm{OR}=0.59(0.25-1.38) \\
P=0.217\end{array}$ \\
\hline Unknown ${ }^{b}$ & 2 & 68 & \\
\hline
\end{tabular}

${ }^{a}$ The $p$-value was either calculated by a chi-square test or a Welch Two Sample t-test. Odds ratios $(\mathrm{OR})$, with confidence intervals of $95 \%(95 \% \mathrm{Cl})$, were calculated ${ }^{\mathrm{b}}$ Has not been added to the calculation. ${ }^{*}$ The $P$-value is significant cNumber of women

\section{Risk factors for Dangme west}

Both age $(p<0.001)$ and parity $(p=0.006)$ (Table 4$)$ were found to be significant risk factors for GBS carriage, while abortion, the level of education, and urinary tract infections were not found to be significant risk factors.

\section{Discussion}

The prevalence of GBS among pregnant women has been studied in many countries; however, very little is known about GBS carriage among pregnant women in developing countries [12]. In recent years, carriage 
Table 2 Serotype distribution for each site

\begin{tabular}{lll}
\hline GBS serotype & $\begin{array}{l}\text { Mamprobi } \\
(\text { Accra })^{\mathrm{b}}\end{array}$ & $\begin{array}{l}\text { Dangme West } \\
\text { (Dodowa) }\end{array}$ \\
$\mathrm{N}=200$ & 200 \\
\hline Ia & 2 & 0 \\
Ib & 0 & 0 \\
II & 1 & 0 \\
III & 2 & 4 \\
IV & 3 & $6^{\mathrm{a}}$ \\
V & 3 & 4 \\
VI & 0 & 0 \\
VII & 24 & 20 \\
VIII & 3 & 4 \\
IX & 18 & $14^{\mathrm{a}}$ \\
Total number & 56 & 51 (52 isolates) $^{\mathrm{a}}$ \\
Percentage carriage & 28.0 & 25.5 .0 \\
& $95 \% \mathrm{Cl}(21.9-34.8)$ & $95 \%$ Cl (19.6-32.1)
\end{tabular}

${ }^{\mathrm{a}}$ One person from Dangme West was positive for two different GBS serotypes, serotype IV and serotype IX

${ }^{\mathrm{b}}$ Number of isolates studies to determine the prevalence and serotype epidemiology among pregnant women have emerged in developing countries. The review study by Dagnew et al. [12] shows an overview of some of the incidence studies performed in developing countries. In the review, the GBS prevalence ranged from $12 \%$ in Asia/Pakistan to $22 \%$ in the Middle East/North Africa. Our study provides GBS prevalence data and serotype distribution for southern Ghana, adding further data to the GBS prevalence in Ghana. A previous study from Ghana presented the prevalence of GBS in the Ashanti region [2] where they found an overall carriage rate of $19.1 \%$, with $23.1 \%$ carriage in Kumasi and 18\% in Pramso. These carriage rates are lower than what is reported at Mamprobi and Dangme West in this study $(28.0 \%$ and $25.5 \%$, respectively) (Table 2). However, both studies showed that the prevalence of GBS is generally high in Ghana. A similar high GBS prevalence has also been observed in studies from other African countries. In a study from Gabon, the GBS carriage rate was reported to be 19\% [13]. Mitima et al. [6] found a GBS prevalence of $20 \%$ in the Congo. Another study showed a carriage rate of $20.2 \%$ in Kenya and 23.1\% in South Africa [14]. In general, all these GBS prevalence studies documented a carriage rate around $20 \%$ or above; however, there are also studies

Table 3 Risk factors for Mamprobi (Accra)

\begin{tabular}{|c|c|c|c|c|c|}
\hline & $\begin{array}{l}\text { GBS carriage } \\
(N=56)\end{array}$ & $\begin{array}{l}\text { No GBS carriage } \\
(N=144)\end{array}$ & OR & $95 \% \mathrm{Cl}$ & $P$ values $^{a}$ \\
\hline \multicolumn{6}{|l|}{ Maternal age group } \\
\hline$<20$ and $>30$ & 29 & 51 & 1 & & \\
\hline $20-30$ & 27 & 92 & 0.52 & $0.28-0.97$ & $P=0.037^{*}$ \\
\hline Unknown ${ }^{\mathrm{b}}$ & 0 & 1 & & & \\
\hline \multicolumn{6}{|l|}{ Parity } \\
\hline 0 & 11 & 32 & 1 & & \\
\hline$>0$ & 38 & 96 & 1.15 & $0.53-2.52$ & $P=0.723$ \\
\hline Unknown ${ }^{\mathrm{b}}$ & 7 & 16 & & & \\
\hline \multicolumn{6}{|l|}{ Abortion } \\
\hline 0 & 42 & 72 & 1 & & \\
\hline$>0$ & 14 & 30 & 0.80 & $0.38-1.68$ & $P=0.554$ \\
\hline Unknown $^{\mathrm{b}}$ & 0 & 42 & & & \\
\hline \multicolumn{6}{|l|}{ Educational level } \\
\hline None or primary & 15 & 39 & 1 & & \\
\hline Junior high school, Senior high school and Post secondary & 40 & 102 & 1.02 & $0.51-2.05$ & $P=0.957$ \\
\hline Unknown $^{\mathrm{b}}$ & 1 & 3 & & & \\
\hline \multicolumn{6}{|l|}{ Urinary tract infections (UTI) } \\
\hline Yes & 3 & 8 & 1 & & \\
\hline No & 52 & 135 & 0.97 & $0.25-3.81$ & $P=0.969$ \\
\hline Unknown ${ }^{\mathrm{b}}$ & 1 & 1 & & & \\
\hline
\end{tabular}

${ }^{\text {TThe }} p$-value was calculated by a chi-square test. Odds ratios (OR) with confidence intervals of $95 \%(95 \% \mathrm{Cl})$ were calculated

${ }^{b}$ Has not been added to the calculation. *The $P$-value is significant 
Table 4 Risk factors for Dangme West (Dodowa)

\begin{tabular}{|c|c|c|c|c|c|}
\hline & $\begin{array}{l}\text { GBS carriage } \\
(N=51)\end{array}$ & $\begin{array}{l}\text { No GBS carriage } \\
(N=149)\end{array}$ & OR & $95 \% \mathrm{Cl}$ & $P$-value ${ }^{a}$ \\
\hline \multicolumn{6}{|l|}{ Maternal age group } \\
\hline$<20$ and $>30$ & 31 & 41 & 1 & & \\
\hline $20-30$ & 20 & 108 & 0.24 & $0.13-0.48$ & $P<0.001^{*}$ \\
\hline Unknown $^{\mathrm{b}}$ & 0 & 0 & & & \\
\hline \multicolumn{6}{|l|}{ Parity } \\
\hline 0 & 0 & 24 & 1 & & \\
\hline$>0$ & 51 & 125 & $10.31^{c}$ & $1.36-78.14$ & $P=0.006^{*}$ \\
\hline Unknown ${ }^{\mathrm{b}}$ & 0 & 0 & & & \\
\hline \multicolumn{6}{|l|}{ Abortion } \\
\hline 0 & 28 & 104 & 1 & & \\
\hline$>0$ & 23 & 45 & 1.90 & $0.99-3.65$ & $P=0.053$ \\
\hline Unknown $^{\mathrm{b}}$ & 0 & 0 & & & \\
\hline \multicolumn{6}{|l|}{ Education level } \\
\hline None or primary & 12 & 44 & 1 & & \\
\hline Junior high school, Senior high school and Post secondary. & 39 & 105 & 1.36 & $0.65-2.84$ & $P=0.41$ \\
\hline \multicolumn{6}{|l|}{ Urinary tract infections (UTI) } \\
\hline Yes & 3 & 12 & 1 & & \\
\hline No & 28 & 120 & 1.10 & $0.28-4.32$ & $P=0.897$ \\
\hline Unknown ${ }^{b}$ & 20 & 17 & & & \\
\hline
\end{tabular}

${ }^{\text {a The }} p$-value is calculated by a chi-square test. Odds ratios (OR), with confidence intervals of $95 \%(95 \% \mathrm{Cl})$, were calculated

${ }^{\mathrm{b}} \mathrm{Has}$ not been added to the calculation. *The $P$-value is significant

'Where zeros cause problems with computation of the odds ratio, 1 was added to all four cells

reporting very low prevalences as seen in the study by Woldu et al. [7], who found a GBS prevalence of $7.2 \%$ in Ethiopia, and de Steenwinkel et al. [15] who reported a GBS prevalence of $1.8 \%$ among pregnant women in Maputo, Mozambique. The reason for the differences in the GBS prevalence between the studies can be attributed to differences in the methodology as suggested by Dagnew et al. [12] In the study by Cools et al. [14], only vaginal swab samples were collected, which they suggest might be responsible for the lower carriage rate, compared to isolation of GBS from both vaginal and rectal swabs. If all the positive rectal swab samples were removed in this study, the carriage rate would have been 24\% for Momprobi and 21\% for Dangme West. This would indicate a reduction in the carriage rate by approximately $4 \%$. It has however also been shown that specimens collected by swabbing the lower vagina and/ or the rectum yield adequate samples for screening [8]. In the study from the Ashanti region in Ghana, they only tested and found five different serotypes, of which serotypes Ia, V, and III were the most common serotypes, particularly in the rural areas [2]. The carriage data that we have collected (Table 2) can be grouped into urban area site (Mamprobi), while Dangme West can be considered a rural site. The findings in this study with respect to GBS serotype distribution are similar to that of Vinnemeier et al. [2] in Kumasi. We furthermore found a similar carriage rate, when comparing the prevalence between rural site (Dangme West, 25.5\% carriage) and urban site (Mamprobi, 28.0\%). It could have been interesting to see whether the five non-typable serotypes from the Vinnemeier et al. [2] study could have been identified as belonging to either serotype VII or IX, if the isolates had been tested for all 10 GBS serotypes. The GBS serotype distribution found at the Mamprobi and Dangme West sites, where serotypes VII and IX were the dominating serotypes at both sites (Table 2), are very different from what has been observed in previous GBS studies in Africa. Several studies from Africa have found that the predominant GBS serotypes were found among the serotypes Ia, Ib, II, III, and serotype V, while serotypes IV, VI, VII, VIII, and serotype IX were not seen $[2,4,16,17]$. In a recent study, however, Cools et al. [14] found a relatively high prevalence of serotypes VI, VII, and VIII in Kenya and serotypes IV, VI and VIII in South Africa, while they did not detect serotype Ib. The reason for these differences in the GBS serotype distribution between the studies from African countries, may be attributed to the geographical location, the typing procedures or other variables [18]. However, testing 
for all ten known serotypes is important, since serotypes normally thought to be prevalent only in certain parts of the world, such as serotype VIII, might also be found at other sites in other parts of the world as seen in the study by Ekelund et al. [19]. In this study, we did not observe any non-typable serotypes (Table 2). It is not uncommon to see studies with no NT isolates [20]. Often NT isolates are found in studies, which do not test for all 10 known serotypes, such as Vinnemeier et al. [20]. We can not explain why we did not find any NT isolates in this study using only serological methods, however because we tested for all ten serotypes, we did reduce the possibilities of NT isolates.

At present, there are two vaccines in phase II trials $[14,18]$, which will cover the serotype Ia, Ib and III. However, as seen in both our studies and other studies $[2,4,14]$, these GBS vaccines would not protect a large number of women in several African countries.

In this study the maternal age was found to be a significant risk factor for both sites, whereas parity was found to be a significant risk factor for Dangme West (Tables 3 and 4). The number of abortions, the level of education, and urinary tract infections were not found to be significant risk factors at any of the study sites. Risk factors for GBS carriage do vary, depending on the study. In a study from DR Congo [6], it was found that age and parity were not risk factors, while education, UTI and abortion were found to be risk factors. In another study from Gabon [13] only illiteracy was found to be a significant risk factor. In the study by Cools et al. [14], they found a statistically significant age-group dependent GBS association in the non-pregnant women, with the lowest and the highest GBS carrier rates in the youngest and the oldest age groups. However, they also found that this age dependent risk could not be seen in pregnant women. According to some studies incidence of invasive disease are increasing in adults with underlying medical conditions such as diabetes mellitus [16]. Diabetes mellitus was therefore investigated as a risk factor, however our data was limited to perform any calculations. Larger GBS carriage studies are needed to give a clearer picture of which risk factors are important in African countries.

\section{Conclusion}

In conclusion, this study found a high prevalence of GBS in pregnant women in Southern Ghana, similar to the prevalence found in other parts of the country. The GBS prevalence is furthermore very similar to other countries in the region and elsewhere in the United States and other Western countries. Regarding serotype distribution, we identified a different serotype distribution pattern in this study compared to what was observed in the study by Vinnemeier et al. [2]. As also observed in the study by Cools et al. [14], the current phase II GBS vaccines focusing only on serotypes Ia, Ib, and III would not protect a large number of the women participating in this study.

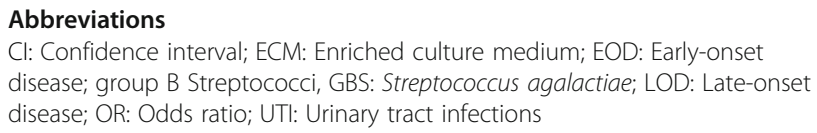

\section{Acknowledgements}

We are grateful to the Danish International Development Assistance (Danida) for providing financial support to the project "Antibiotic Drug Use, Monitoring and Evaluation of Resistance in Ghana" (ADMER) (http://admerproject.org). We also wish to thank the Ghanaian Ministry of Health and the study subjects for their permission to carry out the research. Finally, we are grateful to all senior researchers and their assistants who helped in the Department of Medical Microbiology, School of Biomedical and Allied Health Sciences, University of Ghana, Department of Clinical Microbiology, KNUST, Ghana, Department of Microbiological Surveillance and Research, Statens Serum Institut, Copenhagen for their support of the project.

Ethical approval and consent to paticipate

Ethical approval for the study was obtained from the University of Ghana (SAHS - ET/SAH5/BC/AA/26/2012-2013) and permission to conduct the study was obtained from the Ministry of Education and Health in Ghana. Ethical clearance and approval was obtained from the School of Allied Health Science, the University of Ghana.

\section{Funding}

The Danish International Development Assistance (Danida) has provided financial support to the project "Antibiotic Drug Use, Monitoring and Evaluation of Resistance in Ghana" (ADMER) (http://admerproject.org). This study is a part of the ADMER project.

\section{Availability of data and materials}

The dataset is available upon request.

\section{Authors' contributions}

HCS, NTKDD conceived and designed the study. HCS, NTKDD, JANB, NFM contributed to the protocol writing. JANB collected the clinical samples and conducted the laboratory assays. HCS, NTKDD analyzed the data. HCS, NTKDD, JANB, NFM drafted the manuscript. HCS, NTKDD, JANB, NFM reviewed the data and critically revised the manuscript. All authors have read and approved the final manuscript.

Consent for publication

Not applicable.

Competing interests

The authors declare that they have no competing interests.

\section{Publisher's Note}

Springer Nature remains neutral with regard to jurisdictional claims in published maps and institutional affiliations.

\section{Author details}

'Department of Bacteria, Parasites and Fungi, Statens Serum Institut, Artillerivej 5, -2300 Copenhagen, DK, Denmark. ${ }^{2}$ Department of Medical Microbiology, School of Biomedical and Allied Health Sciences, University of Ghana, Accra, Ghana. ${ }^{3}$ Department of Clinical Microbiology, University Hospital, Hvidovre, Copenhagen, Denmark. 
Received: 12 April 2016 Accepted: 16 July 2017

Published online: 21 July 2017

\section{References}

1. Le Doare K, Heath PT. An overview of global GBS epidemiology. Vaccine. 2013;31(Suppl 4):D7-12. doi:10.1016/j.vaccine.2013.01.009. Review

2. Vinnemeier CD, Brust $P$, Owusu-Dabo E, Sarpong N, Sarfo EY, Bio Y, et al. Group B streptococci serotype distribution in pregnant women in Ghana: assessment of potential coverage through future vaccines. Tropical Med Int Health. 2015;20:1516-24. doi:10.1111/tmi.12589.

3. Schrag SJ, Verani JR. Intrapartum antibiotic prophylaxis for the prevention of perinatal group B streptococcal disease: experience in the United States and implications for a potential group B streptococcal vaccine. Vaccine. 2013; 31(Suppl 4):D20-6. doi:10.1016/j.vaccine.2012.11.056.

4. Belard S, Toepfner N, Capan-Melser M, Mombo-Ngoma G, Zoleko-Manego R, Groger M, et al. Streptococcus agalactiae serotype distribution and antimicrobial susceptibility in pregnant women in Gabon. Central Africa Sci Rep. 2015;5:17281. doi:10.1038/srep17281.

5. Mavenyengwa RT, Afset JE, Schei B, Berg S, Caspersen T, Bergseng H, et al. Group B Streptococcus colonization during pregnancy and maternal-fetal transmission in Zimbabwe. Acta Obstet Gynecol Scand. 2010;89:250-5. doi:10.3109/00016340903398029.

6. Mitima KT, Ntamako S, Birindwa AM, Mukanire N, Kivukuto JM, Tsongo K, et al. Prevalence of colonization by Streptococcus agalactiae among pregnant women in Bukavu, Democratic Republic of the Congo. J Infect Dev Ctries. 2014;8:1195-200. doi:10.3855/jidc.5030.

7. Woldu ZL, Teklehaimanot TG, Waji ST, Gebremariam MY. The prevalence of group B Streptococcus recto-vaginal colonization and antimicrobial susceptibility pattern in pregnant mothers at two hospitals of Addis Ababa. Ethiopia Reprod Health. 2014;11:80. doi:10.1186/1742-4755-11-80.

8. Gupta C, Briski LE. Comparison of two culture media and three sampling techniques for sensitive and rapid screening of vaginal colonization by group B streptococcus in pregnant women. J Clin Microbiol. 2004;42:3975-7.

9. Verani JR, McGee L, Schrag SJ, Division of Bacterial Diseases, National Cente for Immunization and Respiratory Diseases, Centers for Disease Control and Prevention (CDC). Prevention of perinatal group B streptococcal diseaserevised guidelines from CDC, 2010. MMWR Recomm Rep. 2010;59(RR-10):1-36.

10. Slotved HC, Elliott J, Thompson T, Konradsen HB. Latex assay for serotyping of group B Streptococcus isolates. J Clin Microbiol. 2003:41:4445-7.

11. O'Brien $\mathrm{KL}$, Nohynek N. World health organization pneumococcal vaccine trials carriage working group: world health organization pneumococcal vaccine trials carriage working group. Report from a WHO working group: standard method for detecting upper respiratory carriage of Streptococcus pneumoniae. Pediatr Infect Dis J. 2003;22:133-40.

12. Dagnew AF, Cunnington MC, Dube Q, Edwards MS, French N, Heyderman $\mathrm{RS}$, et al. Variation in reported neonatal group B streptococcal disease incidence in developing countries. Clin Infect Dis. 2012;55(1):91-102. doi:10.1093/cid/cis395. Review

13. Capan-Melser M, Mombo Ngoma G, Akerey-Diop D, Basra A, Würbel H, Groger $M$, et al. Evaluation of intermittent preventive treatment of malaria against group B Streptococcus colonization in pregnant women: a nested analysis of a randomized controlled clinical trial of sulfadoxine/pyrimethamine versus mefloquine. J Antimicrob Chemother. 2015;70:1898-902.

14. Cools P, Jespers V, Hardy L, Crucitti T, Delany-Moretlwe S, Mwaura M, et al. A multi-country cross-sectional study of vaginal carriage of group $B$ streptococci (GBS) and Escherichia coli in resource-poor settings: Prevalences and risk factors. PLoS One. 2016;11(1):e0148052. doi:10.1371/journal.pone.0148052.

15. de Steenwinkel FD, Tak HV, Muller AE, Nouwen JL, Oostvogel PM, Mocumbi SM. Low carriage rate of group B Streptococcus in pregnant women in Maputo. Mozambique Trop Med Int Health. 2008;13:427-9. doi:10.1111/j.1365-3156.2008.02018.x.

16. Chukwu MO, Mavenyengwa RT, Monyama CM, Bolukaoto JY, Lebelo SL, Maloba MR, et al. Antigenic distribution of Streptococcus agalactiae isolates from pregnant women at Garankuwa hospital - South Africa. Germs. 2015;5:125-33. doi:10.11599/germs.2015.1080.

17. Madzivhandila M, Adrian PV, Cutland CL, Kuwanda L, Schrag SJ, Madhi SA Serotype distribution and invasive potential of group B Streptococcus isolates causing disease in infants and colonizing maternal-newborn dyads. PLoS One. 2011;6:e17861. doi:10.1371/journal.pone.0017861.
18. Tam PY, Delair SF, Obaro SK. Neonatal group B streptococcus disease in developing countries: are we ready to deploy a vaccine? Expert Rev Vaccines. 2015;14:1401-3. doi:10.1586/14760584.2015.1077121.

19. Ekelund K, Slotved HC, Nielsen HU, Kaltoft MS, Konradsen HB. Emergence of invasive serotype VIII group B streptococcal infections in Denmark. J Clin Microbiol 2003:41:4442-4.

20. Ippolito DL, James WA, Tinnemore D, Huang RR, Dehart MJ, Williams J, et al. Group B Streptococcus serotype prevalence in reproductive-age women at a tertiary care military medical center relative to global serotype distribution. BMC Infect Dis. 2010;10:336.

\section{Submit your next manuscript to BioMed Central and we will help you at every step:}

- We accept pre-submission inquiries

- Our selector tool helps you to find the most relevant journal

- We provide round the clock customer support

- Convenient online submission

- Thorough peer review

- Inclusion in PubMed and all major indexing services

- Maximum visibility for your research

Submit your manuscript at www.biomedcentral.com/submit
C) Biomed Central 